\title{
Changing effects of frontal lesions in man
}

\author{
AARON SMITH \\ From the Neuropsychological Laboratory, Nebraska Psychiatric Institute, Department of Neurology and \\ Psychiatry, University of Nebraska College of Medicine, Omaha, Nebraska, U.S.A.
}

The historical controversy on effects of variously situated brain lesions on mental function reflects inherent difficulties in precise definitions of their onset, locus, magnitude, and dynamics in neurological populations. The introduction of psychosurgery in efforts to relieve chronic psychoses permitted systematic studies of carefully designed frontal lobe lesions. Early findings, however, were as diverse as those reported in studies of brain wounds and tumours (Smith, 1962).

Three related studies initiated in 1946, the Columbia-Greystone studies I and II (Mettler, 1949 and 1952), and the New York State brain research (Lewis, Landis, and King, 1956) projects marked a major advance in controlled study of effects of brain surgery. Increasingly refined research designs included standardized surgical procedures with systematic excisions of different cortical areas, planned sampling, experimental controls, and objective measures of mental function obtained at fixed intervals before and after surgery. Yet, in view of traditional concepts of specific mental defects following frontal lesions, and other studies of similar populations reporting various intellectual deficits, the unanimous negative findings in all three projects were intriguing.

Since initial post-operative decrements in mental tests administered in all three studies were followed by recovery of pre-operative levels within the first year, this 'drop and rise' pattern was cited as evidence that regardless of site, patient's age, or amounts excised (up to a known maximum of $73.5 \mathrm{~g}$.), clean circumscribed frontal ablations did not result in permanent impairment. These unanimously negative findings have been extensively cited in revisions of neurological textbooks and in studies reporting intellectual deficits following non-frontal lesions.

Re-examinations of 28 topectomy and 24 carefully matched control schizophrenics admitted to hospital under the New York State brain research project eight years post-operatively, however, revealed systematic losses by operated patients in several mental tests (Smith, 1958, 1960; Smith and Kinder, 1959). Comparisons of pre-operative, initial, and eight-year post-operative scores indicated that effects of lesions varied with interactions of four factors: time, site, age, and nature of the measure. In contrast to early post-operative findings showing recovery of pre-operative levels and no significant differences between changes by operated and control patients, eight-year post-operative scores showed systematic losses by older and younger patients with posterior frontal excisions (superior topectomy) and by older patients with anterior frontal excisions (orbital topectomy). Younger patients with orbital topectomy and younger and older controls showed only negligible score changes after eight years. Thus the findings of this follow-up study were consistent with many reports, e.g., Holmes (1927), PurvesStewart (1927), Bailey (1948), and Russell (1959), that mental sequelae of frontal tumours and brain wounds varied, depending upon their specific site within the frontal lobes.

The present report summarizes results of a followup study of 31 of 43 operated and 11 of 19 control patients of the two Columbia-Greystone projects who were still in hospital in 1958, 10 to 12 years after surgery. The seven surgical procedures (Mettler, 1949; Mettler, 1952) reduced the numbers in each operated category, precluding statistical analyses of the data. Use of the same (Wechsler-Bellevue I, Porteus maze, homograph, and Weigl sorting) tests, however, provided comparable data for reassessments of the four factors indicated in the follow-up study of the patients concerned in the New York State brain research project.

Table I presents descriptive data with pre-operative raw scores and changes shown in 1958 scores for each of the 31 operated patients arranged in a rank order starting with the patient with the most frequent losses and ending with the patient with no losses. (Although the use of unoperated patients as controls in these studies was prevented by significant differences in prognoses and other variables described later by the original investigators (Mettler, 1949, 1952), mean scores for the 11 unoperated patients were included for gross comparisons. In the light of recurring interest in surgical therapy of chronic psychoses, it might also be pointed out that early discharge of a higher proportion of operated than 
TABLE I

PRE-OPERATIVE RAW SCORES AND CHANGES AFTER POST-OPERATIVE INTERVAL OF 10 TO 12 YEARS (1947-19581)

\begin{tabular}{|c|c|c|c|c|c|c|c|c|c|c|c|c|}
\hline \multirow{3}{*}{$\begin{array}{c}\text { Case } \\
\text { No. }\end{array}$} & \multirow[t]{3}{*}{ Operation $^{2}$} & \multirow[t]{3}{*}{ Age } & \multirow[t]{3}{*}{ Brodmann Areas $^{3}$} & \multirow{3}{*}{$\begin{array}{l}\text { Amount of } \\
\text { Tissue } \\
\text { Excised }\end{array}$} & \multicolumn{7}{|l|}{ Test } & 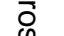 \\
\hline & & & & & \multicolumn{2}{|c|}{ Information } & \multicolumn{2}{|c|}{ Comprehension } & \multicolumn{2}{|c|}{ Digit Span } & \multicolumn{2}{|c|}{ Arithme先 } \\
\hline & & & & & Pre-op. & Change $e^{1}$ & Pre-op. & Change & Pre-op. & Change & Pre-op. & \\
\hline 1 & Topectomy & 58 & Posterior 8, 9, 10, 46 & 38.4 more & 11 & -7 & 6 & $\mathbf{0}$ & 9 & -1 & 3 & \\
\hline 2 & Topectomy & 32 & Posterior $8,9,10$ & 29.8 more & 19 & -3 & 18 & -5 & 14 & -2 & 10 & \\
\hline 3 & Thermocoagulation & 30 & Posterior 9,10 & See note & 9 & -1 & 8 & -4 & 8 & -2 & 7 & \\
\hline 4 & Topectomy & 51 & Posterior 9 & $33 \cdot 1$ more & 9 & -2 & 12 & -7 & 9 & -1 & 3 & i: \\
\hline 5 & Thalamotomy & 32 & Dorso-medial nucleus & - & 13 & -5 & 5 & 0 & 9 & -1 & 7 & $\overline{\bar{\omega}}-$ \\
\hline 6 & Topectomy & 30 & Posterior 8, 9, 10, 46 & 30 more & 17 & -3 & 10 & -4 & 9 & -1 & 6 & $\frac{\pi}{2}$ \\
\hline 7 & Venous ligation & 34 & - & - & 14 & -6 & 9 & -4 & 10 & -1 & 7 & \\
\hline 8 & Topectomy & 32 & Posterior 6, 8 & 24.9 more & 15 & -1 & 3 & 4 & 9 & $\mathbf{0}$ & 5 & 인 \\
\hline 9 & Venous ligation (limited) & 52 & - & - & 14 & -1 & 11 & $\mathbf{0}$ & 11 & 3 & 7 & \\
\hline 10 & Transorbital lobotomy & 45 & - & - & 13 & -2 & 3 & $\mathbf{0}$ & 9 & $\mathbf{0}$ & 6 & $\frac{1}{n}$ \\
\hline 11 & Topectomy & 53 & Anterior $10,11,45,46,47$ & 73.5 most & 10 & -6 & 8 & 1 & 8 & $\mathbf{0}$ & 5 & \\
\hline 12 & Venous ligation & 51 & - & - & 12 & 2 & 9 & -2 & 8 & $\mathbf{0}$ & 8 & \\
\hline 13 & Venous ligation (limited) & 32 & - & - & 19 & -4 & 5 & 1 & 12 & 2 & 5 & - \\
\hline 14 & Venous ligation (limited) & 32 & - & 一 & 6 & -2 & 9 & -2 & 8 & 2 & 4 & \\
\hline 15 & Venous ligation (limited) & 36 & - & - & 12 & -1 & 8 & 3 & 10 & -2 & 5 & 5 \\
\hline 16 & Transorbital lobotomy & 30 & - & - & 8 & -2 & 3 & 0 & 9 & $\mathbf{0}$ & 2 & $\perp$ \\
\hline 17 & Prefrontal lobotomy & 27 & - & - & 4 & --4 & 1 & 2 & 7 & $\mathbf{0}$ & $\mathbf{0}$ & 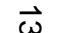 \\
\hline 18 & Topectomy & 31 & Anterior 44 & N.A. (least) & 9 & -3 & 5 & -2 & 9 & $\mathbf{0}$ & 3 & w \\
\hline 19 & Venous ligation & 47 & - & - & 9 & 3 & 8 & 5 & 9 & 2 & 7 & \\
\hline 20 & Transorbital lobotomy & 33 & - & - & 8 & -2 & 5 & 5 & 10 & -1 & 2 & \\
\hline 21 & Topectomy & 39 & Anterior 11 & 26 least & 22 & -2 & 18 & -3 & 13 & 2 & 10 & 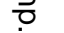 \\
\hline 22 & Prefrontal lobotomy & 39 & - & - & 20 & $\mathbf{0}$ & 14 & 1 & 10 & 1 & 11 & No \\
\hline 23 & Venous ligation & 33 & - & - & 12 & 3 & 11 & -1 & 7 & 1 & $\mathbf{0}$ & \\
\hline 24 & Transorbital lobotomy & 33 & - & - & 6 & -1 & 2 & 5 & 8 & $\mathbf{0}$ & 2 & ه \\
\hline 25 & Thalamotomy & 26 & Dorso-medial nucleus & - & 12 & 2 & 5 & $\mathbf{0}$ & 4 & 5 & 20 & rن \\
\hline 26 & Venous ligation & 33 & - & - & 12 & 3 & 5 & 3 & 10 & -1 & 60 & \\
\hline 27 & Venous ligation (limited) & 26 & - & - & 12 & 4 & 5 & 2 & 10 & 2 & $\frac{6}{8}$ & \\
\hline 28 & Thermocoagulation & 30 & Posterior 9, 10 & 一 & 9 & $\mathbf{0}$ & 2 & 1 & 8 & 2 & 5 & 음- \\
\hline 29 & Transorbital lobotomy & 34 & - & - & 4 & 5 & 2 & 0 & 5 & 3 & $\vec{D}$ & + \\
\hline 30 & Transorbital lobotomy & 27 & - & - & 9 & 2 & 2 & 2 & 9 & 1 & 3 & \\
\hline 31 & Prefrontal lobotomy & 28 & - & - & 11 & $\mathbf{0}$ & 2 & 4 & 8 & 0 & 10 & \\
\hline
\end{tabular}

'Change after 10-12 years. Weigl sorting tests yielded dichotomous scores, i.e., a patient either could or could not shift the bases of sorting frgm colour or vice versa. Patients ranked 1 to 13 included seven able to shift pre-operatively, five of whom were unable to shift 10 to 12 years latescerm to a similar loss by only one of six for ranks 14-31. Three of 11 controls were able to shift initially and two of these three were able to shift 10 fo 2 later. Changes in other scores for the 11 controls were: Information $12.91(-0.27)$; comprehension 8.82 ( -0.37$)$; digit span 10. $₫$ ( $₹$. arithmetic $6.45(-0.18)$; similaritięs $9.73(-0.36)$; vocabulary $23.18(-1.09)$; picture arrangement $7 \cdot 18(0.37)$; picture completion $8.36(0.37)$; bäpct广, 15.27 (-1.54); object assembly 12.73 (2.91); digit symbol 24.55 (-1.05); Porteus maze 9.75 (-0.95); homographs 12.09 (-0.91).

2 Operative procedures and actual amounts of tissue excised are described by Mettler (1949, 1952). Surgical estimates of amounts of tissue rendef functional (least, more, most) were considered more reliable though less precise than estimated weights in grams.

sPosterior is caudal to Brodmann's area 10 and anterior is rostral to Brodmann's area 10.

-Thermocoagulation for 25 seconds longer than case 28.

of unoperated patients was later attributed to significant prognostic differences. Despite these differences, however, the overall results of the three projects showed no significant differences in discharge rates of operated and unoperated patients.)

Retests of operated patients within one year of surgery showed recovery of pre-operative levels. Effects of time are thus indicated in losses over 10 years later by patients nos. 1 to 13 . Systematic differences in interactions of time with site, age, mass, and the nature of measures showing marked decrements indicate that losses were due to long-term effects of surgery rather than to mental deterioration in chronic psychotic in-patients.

Eight topectomy cases were arbitrarily divided into anterior (excisions rostral to Brodmann's area 10) and posterior (caudal to Brodmann's area 10) groups. The more marked losses by the posterior group 12 years post-operatively confirms King (1949) observations of larger initial losses followi g excisions of Brodmann area 8, although, as noted above, subsequent tests administered within the figt year indicated recovery.

Similarly, more marked losses by older patients in each type of operative procedure also corresponded to the findings of the New York State bram research project follow-up study reflecting the s: nificance of the age factor.

Differences in the sensitivity of the various measures were also consistent with the same followup study. Digit symbol, block design, homograph, and information scores showed the most frequent and marked losses. Decrements in the Porteus ma䆣, arithmetic, and digit span tests were less frequent and marked in the present study. Thus the differef tial sensitivity of the various tests in both follow- 
TA BLE I-continued

PRE-OPERATIVE RAW SCORES AND CHANGES AFTER POST-OPERATIVE INTERVAL OF 10 TO 12 YEARS (1947-19581)

\begin{tabular}{|c|c|c|c|c|c|c|c|c|c|c|c|c|c|c|c|c|c|}
\hline \multicolumn{2}{|c|}{ nilarities } & \multicolumn{2}{|c|}{ Vocabulary } & \multicolumn{2}{|c|}{$\begin{array}{l}\text { Picture } \\
\text { Arrangement }\end{array}$} & \multicolumn{2}{|c|}{$\begin{array}{l}\text { Picture } \\
\text { Completion }\end{array}$} & \multicolumn{2}{|l|}{$\begin{array}{l}\text { Block } \\
\text { Design }\end{array}$} & \multicolumn{2}{|c|}{$\begin{array}{l}\text { Object } \\
\text { Assembly }\end{array}$} & \multicolumn{2}{|l|}{$\begin{array}{l}\text { Digit } \\
\text { Symbol }\end{array}$} & \multicolumn{2}{|c|}{$\begin{array}{l}\text { Porteous } \\
\text { Maze }\end{array}$} & \multicolumn{2}{|c|}{ Homographs } \\
\hline$\therefore o p$ & Change & Pre-op. & Change & Pre-op. & Change & Pre-op. & Change & Pre-op. & Change & Pre-op. & Change & Pre-op. & Change & Pre-op. & Change & Pre-op. & Change \\
\hline 8 & -1 & 12 & -2 & 4 & -4 & 7 & -.6 & 11 & -11 & 16 & -10 & 12 & -12 & 13 & -5.5 & 13 & -3 \\
\hline 20 & -3 & 30 & $1 \cdot 5$ & 16 & $\ldots 7$ & 13 & -4 & 25 & -5 & 25 & -4 & 43 & -10 & 11 & 2 & 26 & -15 \\
\hline 7 & -4 & 10 & -0.5 & 9 & -5 & 3 & 2 & 6 & 6 & 11 & -4 & 15 & -10 & 4 & 0.5 & 6 & -2 \\
\hline 12 & -7 & 14 & $0 \cdot 5$ & 6 & 0 & 8 & 0 & 14 & -5 & 16 & -4 & 26 & -6 & $11 \cdot 5$ & $-4 \cdot 5$ & 13 & -2 \\
\hline 4 & 0 & $17 \cdot 5$ & -10 & 6 & -2 & 8 & -2 & 3 & 12 & 15 & 3 & 27 & -6.5 & 6 & -0.5 & 4 & -3 \\
\hline 10 & 2 & 26 & -3 & 11 & -4 & 8 & 0 & 15 & 2 & 11 & 6 & 43 & -11 & 15 & $-4 \cdot 5$ & 14 & -5 \\
\hline 15 & -6 & 21 & 3.5 & 9 & -3 & 7 & 3 & 7 & 1 & 10 & 4 & $20 \cdot 5$ & $3 \cdot 5$ & 6 & 0.5 & NA & \\
\hline 13 & -1 & 21 & -0.5 & 4 & 2 & 12 & 2 & 24 & -2 & 17 & $\ldots 1$ & 31 & 7 & 15 & -0.5 & 8 & 10 \\
\hline 12 & --2 & $21 \cdot 5$ & 0 & 6 & 4 & 10 & $\cdots 1$ & 12 & 6 & 17 & 5 & 37 & -1 & 8 & -2 & 11 & -3 \\
\hline 3 & -3 & 16 & -6 & 0 & 2 & 7 & 0 & 0 & 0 & 14 & -8 & 12 & 12 & $6 \cdot 5$ & 0.5 & 1 & -1 \\
\hline 11 & -4 & 17 & 0.5 & 4 & 0 & 9 & 1 & 17 & -14 & 15 & 1 & 20 & $\ldots 12$ & 10 & 1 & 18 & -5 \\
\hline 6 & 2 & $19 \cdot 5$ & $-1 \cdot 5$ & 5 & 1 & 6 & 2 & 12 & -9 & 14 & 2 & $20 \cdot 5$ & -15.5 & NA & & 11 & -1 \\
\hline 12 & -12 & 26 & -4.5 & 7 & 2 & 14 & -1 & 21 & 6 & 18 & 3 & 19 & 2 & $12 \cdot 5$ & -2 & 11 & 0 \\
\hline 10 & 2 & $14 \cdot 5$ & -2 & 10 & 1 & 5 & 3 & 14 & $\cdots 1$ & 18 & $i$ & 35 & 0 & 7 & 4 & 7 & 5 \\
\hline 3 & 6 & $18 \cdot 5$ & $1 \cdot 5$ & 8 & 3 & 5 & 1 & 16 & 0 & 14 & 6 & $28 \cdot 5$ & -5 & 7 & 0 & 13 & 2 \\
\hline 7 & 1 & $17 \cdot 5$ & $1 \cdot 5$ & 6 & 3 & 8 & -1 & 12 & 4 & 16 & 2 & 18 & 8 & 16 & -5 & 7 & $\ldots \overline{1}$ \\
\hline 0 & 5 & 0 & $6 \cdot 5$ & 0 & 0 & 9 & -4 & 9 & 0 & 12 & 4 & 5 & 7 & 7 & $-1 \cdot 5$ & 2 & -2 \\
\hline 8 & 3 & 23 & -5.5 & 2 & 2 & 7 & $\ldots 1$ & 12 & 0 & 15 & 3 & 11 & $9 \cdot 5$ & 6 & $5 \cdot 5$ & 5 & $\overline{1}$ \\
\hline 7 & 0 & 21 & 0 & 1 & 9 & 9 & -1 & 12 & 5 & 14 & 5 & 21 & 2 & $11 \cdot 5$ & $-3 \cdot 5$ & 10 & 3 \\
\hline 2 & 2 & 13.5 & -1.5 & 0 & 6 & 3 & 4 & 6 & '0 & 14 & 5 & 21 & 5 & 6 & 0.5 & 5 & -3 \\
\hline 7 & 2 & 33 & 2 & 18 & -4 & 13 & 0 & 32 & 1 & 21 & 2 & 36 & 1 & 18 & 0 & 21 & 2 \\
\hline 8 & 0 & 34 & 2 & 9 & -3 & 14 & -1 & 30 & 5 & 21 & 3 & 24 & 9 & 14 & $3 \cdot 5$ & 27 & 4 \\
\hline 7 & 1 & 26 & 1 & 9 & -1 & 7 & 5 & 14 & 4 & 20 & 0 & 19 & 7 & 7 & -1 & 6 & 9 \\
\hline 2 & 7 & 13.5 & $-2 \cdot 5$ & 0 & 2 & 2 & 1 & 0 & 0 & 6 & 0 & 0 & $20 \cdot 5$ & $4 \cdot 5$ & -0.5 & 3 & 0 \\
\hline 4 & 1 & $7 \cdot 5$ & $4 \cdot 5$ & 0 & 4 & 4 & -2 & 3 & 4 & 8 & -4 & 26 & 0 & $13 \cdot 5$ & -8 & 0 & 0 \\
\hline 6 & -7 & 21 & 1 & 7 & 3 & 11 & 1 & 16 & 1 & 15 & 5 & 15 & $5 \cdot 5$ & $9 \cdot 5$ & 1 & 9 & 0 \\
\hline 4 & 6 & $19 \cdot 5$ & 15 & 7 & 1 & 5 & 1 & 9 & 7 & 18 & 0 & 41 & -6 & $7 \cdot 5$ & $2 \cdot 5$ & 12 & $\ldots 5$ \\
\hline 0 & 6 & 14.5 & $2 \cdot 5$ & 4 & 0 & 5 & 0 & 0 & 6 & 11 & 3 & 17 & 6 & $4 \cdot 5$ & 1 & 7 & $\cdots 3$ \\
\hline 5 & 3 & 15 & 2.5 & 0 & 0 & 4 & 0 & 0 & 0 & 7 & -2 & 0 & 5 & NA & & $i$ & 5 \\
\hline 0 & 6 & 12 & 4 & 5 & 8 & 6 & 2 & 24 & 11 & 22 & 3 & 8.5 & $21 \cdot 5$ & 15.5 & 0 & 9 & -4 \\
\hline 4 & 3 & 6 & $14 \cdot 5$ & 2 & 0 & 7 & 2 & 0 & 13 & 0 & 14 & 4 & 8 & $7 \cdot 5$ & 0.5 & 5 & 5 \\
\hline
\end{tabular}

studies reflected the significance of the nature of the measure in assessments of mental sequelae of brain lesions.

Although amounts excised in topectomies in the New York State brain research series were standardized (approximately $30 \mathrm{~g}$. from each frontal lobe), varying amounts of cortical excisions in the two series of patients from Columbia-Greystone provided evidence of the significance of a fifth factor, mass. Since tissue rendered dysfunctional by operative procedures was part of the mass factor, neurosurgical estimates of total amounts (in terms of least, more, most) as well as weights of tissue excised are shown for the eight topectomy cases.

Recovery of pre-operative levels within the first year was unaffected by differences in mass. Longterm losses by patients with larger excisions therefore indicate interactions of time and mass factors. Although the eight topectomy cases include interactions of age and site factors, the significance of mass is strikingly illustrated in comparisons of two 30-yearold patients with thermocoagulation of Brodmann areas 9 and 10. Adhesion of the thermode for 25 seconds longer in patient no. 3 resulted in loss of more cortical tissue and is reflected in losses in 11 measures compared with losses in only two measures by patient no. 28 . Since both patients showed slight gains in post-operative tests in the first year, changes in scores 10 years later are consistent with interactions of time and mass illustrated in the eight topectomy cases.

Interactions of mass and site are illustrated in patients nos. 1, 2, 11, and 21. Although patients nos. 11 and 21 both had anterior lesions, differences in mass $(73.5 \mathrm{~g}$. versus $26.0 \mathrm{~g}$.) not reflected in early post-operative scores are clearly evident in Table 1 . However, excisions in patients no. $1(38.4$ g.) and no. $2(29.8 \mathrm{~g}$.) were approximately half that in patient no. $11(73.5 \mathrm{~g}$.). The greater losses by patients nos. 1 and 2, despite approximately $50 \%$ less tissue excised, thus indicate that site of excision was a more significant determiner of long-term losses than the amount excised. However, the absence of marked losses in patient no. 28 with thermocoagulation of less posterior tissue than patient no. 3 suggests the existence of a mass threshold.

Thus, the overall results confirm the findings of the follow-up study of the patients in the New York State brain research project. In both studies, systematic differences between initial and long-term 
effects of brain lesions on mental functions reflected interactions of time with the factors of site, age, and nature of the measure. The emergence of long-term losses by patients with larger lesions in the present study indicates that mass is a fifth significant factor.

Since losses occurred in measures involving different modalities, the defect appears to be an impairment of higher mental functions. This defect occurred most frequently after bilateral posterior lesions regardless of age; in older patients regardless of site; and in patients with larger lesions regardless of site or age. When these factors are taken into account, differences as a function of type of surgery appear negligible.

In the absence of pathological anatomy, possible anatomical bases for the differences between early and long-term effects of frontal ablations remain undefined. However, systematic differences in psychological test performances as a function of time, site, and age are consistent with findings of clinical studies, and are strikingly consonant with neuropathological studies of psychosurgical patients describing increasing secondary degeneration with time, age, and in posterior frontal lesions (Smith, 1962).

\section{DISCUSSION}

Since over half the population revealed little evidence of mental deterioration, viewed in the wide context of the voluminous pertinent literature, the findings as a whole might not appear to have had any definite outcome. The emergence of systematic losses by patients with posterior lesions, and regardless of site, by patients with advanced age or larger anterior lesions, however, clearly indicates that traditional comparisons of undifferentiated 'brain-damaged' populations are methodologically unwarranted. Since losses were not present shortly after surgery, time is one of the most important factors in assessments of brain lesions.

The post-operative interval of 10 to 12 years is almost identical with the post-injury interval in the follow-up study of brain-wounded war veterans by Weinstein and Teuber (1957). Since losses in intelligence test scores were reported only for the small group with left temporo-parietal wounds, this study has been frequently cited as evidence that non-frontal lesions result in greater deficits than frontal lesions. A recent review by Piercy (1964) cited the negative early post-operative findings of the first ColumbiaGreystone project and other psychosurgical studies in contrast to the findings of Weinstein and Teuber after 10 to 12 years in support of this view.

In addition to the questionable validity of comparing populations with ill-defined heterogeneous lesions of different ages, Smith (1962) has noted that comparisons of groups differentiated according to lobar, hemispheric, frontal versus non-frontal $\bar{z}$ cortical versus subcortical, and infra- versus supra $\mathbb{Q}$ tentorial lesions tacitly assume a functional equio potentiality of all areas comprising these arbitrarilye defined gross anatomical structures. Before Wein stein and Teuber (1957), Schiller (1947) also reported that as wounds encroached further into a left 'non frontal' region (actually up and along the slant of the Sylvian fissure), both language and intellectua $\vec{E}$ defects became more marked. Bailey (1948) has observed that in addition to specifically localized? tumours of the frontal lobes, intellectual deficit $\bar{Q}$ were more frequent in patients with left occipito temporal tumours. And in Russell's (1963) recen considerations of anatomical aspects of aphasia, hi $\$$ formulations of the dynamic relationships between intra- and interhemispheric brain mechanisms emphasized definitions of the differing roles of various brain structures in lower and higher cerebra $\vec{\omega}$ functions.

At least 14 studies (Smith, 1962), however, have consistently reported marked differences in anatom? ical and functional sequelae of carefully designed frontal ablations with significantly greater intellectuaps impairment following posterior frontal lesions. In addition to the apparent significance of the specific site of brain lesions as one of several significant factors, the results of the present study confirm the importance of age in evaluations of brain injuries $\mathbb{D}$ Since Russell (1934) concluded that age was onerof the most important factors in such evaluations, ivivis hardly surprising that the patient with the largest and most losses among the 31 patients with front lesions was the oldest, albeit, as noted above, these losses reflect interactions of several factors.

As in the follow-up study of the New Yorko State brain research project, the results of the presents follow-up study reverse the original negative find ings reported shortly after surgery. The specific findings in frontal lobe lesions also have methodo logical implications for investigations of othe $\overrightarrow{\overrightarrow{0}}$ lesions which may be summarized.

TIME Differences between initial and long-term findings clearly indicate that initial effects of brain lesions are often transient. In some cases, early? mental impairment may gradually disappear. Iñ others with no evidence of early impairment, defects. may emerge with the passage of time. Since the findings of examinations at different historica $\bar{B}$ periods after brain lesions vary with the dynamics of the lesion, initial assessments will often be least reliable.

SPECIFIC SITE Differences between effects of bi lateral posterior and comparable anterior lesion\$ 
within the frontal lobes, and similar differences in lateralized lesions in other gross structures emphasize the importance of precise definitions of the specific site of lesions as a crucial factor in assessments of effects on mental as well as on other functions.

AGE Since the same lesions in older patients resulted in more frequent and marked impairment than that in younger patients, age is one of the most important determiners of mental as well as other sequelae of brain injuries.

NATURE OF THE DEFECT The different modalities and the mental functions involved in tests of higher intellectual capacities are variously vulnerable to effects of differently situated lesions. The nature and degree of observed impairment will therefore vary, depending upon the selection of tests used and the specific site of the lesion. Since losses following bilateral frontal ablations occurred in tests involving different modalities, the resulting defect appears to be a loss in 'higher' gnostic and conceptual functions. Thus, the validity of definitions of mental impairment is improved in increasing differentiations of impairment of test modalities from impairment of the 'higher' functions the tests were assumed to measure.

SIZE OF LESION Although small and large lesions may reveal no early mental defects, the emergence of later defects is more likely in patients with larger lesions. Thus, while all patients should be reexamined for the possible emergence of later defects, conclusions of no mental deficits in patients with large brain lesions are especially qualified by the absence of long-term reassessments.

The present study confirms the view that as time passes mental deterioration appears in adults with specific frontal lesions produced by operations for mental disorder. These findings are also of interest from the point of view of understanding cerebral mechanisms underlying the development and maintenance of intelligence. Russell (1959) has pointed out that the development of intelligence is markedly attenuated by frontal lobe lesions in infancy. Since specific frontal lobe lesions in adults interfere with the maintenance of intellectual efficiency to a greater degree than lesions of the same size in other frontal and non-frontal areas, the findings of the present study indicate that the integrity of certain structures within the frontal lobes is essential not only for the development but also for the maintenance of intelligence.

\section{SUMMARY}

Psychological re-examinations of 31 psychotic in-patients 10 to 12 years after psychosurgery revealed the emergence of losses by operated groups as a function of time, site, amount excised, age, and nature of the measure. The results confirm findings of the New York State brain research follow-up study and illustrate the varying dynamics of different brain lesions in effects on mental functions.

This study was made possible by the foresight of Dr. Elaine F. Kinder, U.S.P.H.S. Research Grant M-1191, with partial support of U.S.P.H.S. research grant HD-00370, and of the Research Foundation for Mental Hygiene, New York State Department of Mental Hygiene. The kind cooperation of Dr. F. A. Mettler and Dr. A. Crandall is also gratefully acknowledged.

\section{REFERENCES}

Bailey, P. (1948). Intracranial Tumours, 2nd ed. Blackwell, Oxford. Holmes, G. (1927). Discussion on changes in personality in tumours of the frontal lobe. Brain, 50, 485-486.

King, H. E. (1949). In Selective Partial Ablation of the Frontal Cortex edited by F. A. Mettler, pp. 178-207. Hoeber, New York.

Lewis, N. D. C., Landis, C., and King, H. E. (1956). Studies in Topectomy. Grune and Stratton, New York.

Mettler, F. A. (1949). Selective Partial Ablation of the Frontal Cortex. Hoeber, New York.

(1952). Psychosurgical Problems. Blakiston, Philadelphia.

Piercy, M. (1964). The effects of cerebral lesions on intellectual function: a review of current research trends. Brit. J. Psychiat. $110,310-352$.

Purves-Stewart, J. (1927). Intracranial Tumours and Some Errors in their Diagnosis. Oxford University Press, London.

Russell, W. R. (1934). The after-effects of head injury. Trans. med. chir. Soc. Edinb., 1933-4, 48, 129-141.

(1959). Brain, Memory, Learning. Oxford University Press, London.

(1963). Some anatomical aspects of aphasia. Lancet, 1, 1173-1177.

Schiller, F. (1947). Aphasia studied in patients with missile wounds. J. Neurol. Neurosurg. Psychiat., 10, 183-197.

Smith, A. (1958). Changes in Psychological Test Performances of Brain-operated Schizophrenics after an Eight-year Interval. Unpublished doctoral dissertation, Yeshiva University, New York. (1960). Changes in Porteus maze scores of brain-operated schizophrenics after an eight-year interval. J. ment. Sci., 106, 967-978.

- (1962). Ambiguities in concepts and studies of 'brain damage' and organicity. J. nerv. ment. Dis., 135, 311-326.

- and Kinder, E. F. (1959). Changes in psychological test performances of brain-operated schizophrenics after 8 years. Science, 129, 149-150.

Weinstein, S., and Teuber, H. L. (1957). Effects of penetrating brain injury on intelligence test scores. Ibid., 125, 1036-1037. 